\title{
Effects of high-color-discrimination capability spectra on color-deficient vision
}

\author{
Esther Perales, ${ }^{1, *}$ João Manuel Maciel Linhares, ${ }^{2,3}$ Osamu Masuda, ${ }^{3}$ Francisco M. Martínez-Verdú, ${ }^{1}$ and \\ Sérgio Miguel Cardoso Nascimento ${ }^{3}$ \\ ${ }^{1}$ University Institute of Physics Applied to Sciences and Technologies, University of Alicante, 03690 Alicante, Spain \\ ${ }^{2}$ Faculty of Science and Technology, Anglia Ruskin University, Cambridge, UK \\ ${ }^{3}$ Centre of Physics, Campus de Gualtar, University of Minho, 4710-057 Braga, Portugal \\ ${ }^{*}$ Corresponding author: esther.perales@ua.es
}

Received May 2, 2013; revised July 3, 2013; accepted July 22, 2013;

posted July 23, 2013 (Doc. ID 189919); published August 15, 2013

\begin{abstract}
Light sources with three spectral bands in specific spectral positions are known to have high-color-discrimination capability. W. A. Thornton hypothesized that they may also enhance color discrimination for color-deficient observers. This hypothesis was tested here by comparing the Rösch-MacAdam color volume for color-deficient observers rendered by three of these singular spectra, two reported previously and one derived in this paper by maximization of the Rösch-MacAdam color solid. It was found that all illuminants tested enhance discriminability for deuteranomalous observers, but their impact on other congenital deficiencies was variable. The best illuminant was the one derived here, as it was clearly advantageous for the two red-green anomalies and for tritanopes and almost neutral for red-green dichromats. We conclude that three-band spectra with high-colordiscrimination capability for normal observers do not necessarily produce comparable enhancements for color-deficient observers, but suitable spectral optimization clearly enhances the vision of the color deficient. () 2013 Optical Society of America

OCIS codes: (330.1690) Color; (330.1720) Color vision; (330.1730) Colorimetry; (330.6180) Spectral

discrimination.

http://dx.doi.org/10.1364/JOSAA.30.001780
\end{abstract}

\section{INTRODUCTION}

In spite of color constancy, i.e., the ability of the visual system to mitigate the visual effects of lighting with different spectral compositions (for a review on this topic, see [1]), the appearance of individual objects or complex scenes is influenced by the spectrum of the illumination. Although these effects can be small for the range of natural daylights (see Table 1 in [1]]), they can be considerable with artificial lights and even impair discrimination [2,3]. In practical applications, these aspects are addressed by color rendering, which is normally equated in relation to fidelity, appeal or preference, and color discrimination []].

High color discrimination is required in many practical tasks, e.g., surveillance and quality control, and was one of the main issues addressed in the classical research of Thornton [5-7]. The capability of light to produce discriminable colors was first evaluated by the color-discrimination index based on the gamut areas expressed in the CIE 1960 UCS chromaticity diagram defined by a small set of illuminated standard colored samples [6-8]. The gamut spanned by an illuminant was adopted in defining other indices to represent similar properties $[\underline{9}, \underline{10}]$. With the same intention, the color rendering capability of a light was introduced by $\mathrm{Xu}$ [11] as a measure of the capability of the light for revealing many different colors and was evaluated by the color volume enclosed by the optimal colors expressed in the three-dimensional space CIE $1976 L^{*} u^{*} v^{*}$. The optimal colors represent the theoretical limits of all colors arising only by reflection (or transmission) [12], and the corresponding loci was computed in a color diagram $[13,14]$ and was recalculated later by MacAdam $[15,16]$ to obtain the MacAdam limits or Rösch-MacAdam limits. The Rösch-MacAdam solid expressed in the CIELAB color space is a powerful tool and has been routinely used to estimate the color rendering capability of daylight and of standard illuminants [17-19], the gamut of color-deficient observers [20], and the theoretical limits of the number of distinguishable colors [21,22]. The strength of this approach to evaluate the extent of discrimination is its generality, since it does not require the use of a set of samples or of a reference illuminant for the calculations.

Thornton studied several properties of spectrally structured sources and suggested a hypothetical white lamp with just three spectral bands (450, 540, and $610 \mathrm{~nm})$ [8] and a more purplish one, also with three spectral bands (430, 530, and $660 \mathrm{~nm}$ ), as producing large gamut areas [6,7]. These wavelengths were called prime colors (for a review on prime colors, see [23]). These triband light sources were considered to enhance color discrimination for the normal color observer, and it was suggested [7] that it may be expected that they do the same for color-deficient observers. Studies based on standard illuminants $[24,25]$ and on the effects of colored filters [26] suggest that some spiky spectra may in fact improve color discrimination for some classes of color-deficient observers, but the spectra with high-color-discrimination capability were not yet systematically tested.

The main goal of this paper was to test the hypothesis that lighting with high-color-discrimination capability for normal observers also improves discrimination for congenital 
color-deficient observers. Three high-color-discrimination illuminants were tested: one suggested by Thornton [6,7], the prime-color illuminant, with spectral bands at 430, 530, and $660 \mathrm{~nm}$; one with peaks at both ends of the visible band and at around $510 \mathrm{~nm}$, which was proved to be $25 \%$ better than daylight and 35\% better than Thornton's prime-color illuminant [27]; and one derived here by maximization of the RöschMacAdam color solid. The methodology to assess the effects on color-deficient observers was to compute and compare the Rösch-MacAdam color volumes rendered by these illuminants for dichromatic and anomalous trichromatic vision.

\section{METHODS}

\section{A. Illuminants}

Two types of illuminants were used in this study: CIE illuminants and illuminants with high-color-discrimination capability. The CIE illuminants [28] included $D$ illuminants with correlated color temperature (CCT) in the range of 25,000 $4000 \mathrm{~K}$ synthesized from Judd's daylight spectral basis functions [29], illuminant A, C, D65, E, F11, FL3.7, and HP1. The high-color-discrimination illuminants included two reported before: the prime-color illuminant derived by Thornton [6,7], with spectral bands at 430,530, and $660 \mathrm{~nm}$, and the one derived by systematically computing the volume of the Rösch-MacAdam solid obtained with sets of metamers with chromaticities at and around the Planckian locus, whose spectral bands had three peaks at both ends of the visible band and at $510 \mathrm{~nm}$ and the chromaticity CIE 1931 (0.32, 0.34) [27]. Hereafter, this illuminant is called Masuda and Nascimento's. The third high-color-discrimination illuminant was derived in the context of the present work by spectral optimization with the methodology described below (see Fig. 1).

\section{B. Optimal Colors and Rösch-MacAdam Volumes}

The optimal colors under each illuminant were obtained by the method proposed by Li et al. [19]. The method consists of a linear programming approach and was preferred in relation to other more complex methods [17] due to its reduced computation time, an important requirement in the optimization procedure applied here. The spectral range was from 380 to $780 \mathrm{~nm}$ with a spectral sampling of $10 \mathrm{~nm}$. For the normal observer, the $X Y Z$ tristimulus values were computed using the CIE 1931 color-matching functions and transformed into the CIELAB color space. For color-deficient observers, the procedure is specified bellow. The $L^{*}$ planes were computed in the range of 10-90 in steps of 10; the planes corresponding to $L^{*}=1$ and $L^{*}=99$ were also computed. For the RöschMacAdam color solid, the number of discernible colors was estimated by calculating the volume because of the direct proportionality between the two quantities $[\underline{17}, \underline{20}, \underline{22}]$. The convex hull volume of the Rösch-MacAdam color solid was then estimated by the MATLAB convhulln function, which is based on the Qhull algorithm [30].

\section{Color-Deficient Observers}

To estimate the effects of the illuminants on color-deficient observers, the color volumes for abnormal color vision were computed using models simulating for normal trichromatic observers the appearance for dichromats and anomalous trichromats (for a detailed discussion of these models, see [20]).

For dichromats, the method is based on assumptions concerning the hues that appear the same to dichromats and normal trichromats [31]. The algorithm represents the color stimuli in $L M S$ space and replaces each stimulus by its projection $L^{\prime} M^{\prime} S^{\prime}$ onto a reduced stimulus surface defined by a neutral axis and by the LMS locations of those monochromatic stimuli that are perceived as the same by normal trichromats and a given type of dichromat. Smith and Pokorny's fundamentals [32] were used to compute cone excitations. This set of fundamentals was based on a transformation of Judd's modification of the color-matching functions of the $1931 \mathrm{CIE}$ standard observer. Thus, the optimal tristimulus values $X Y Z$ derived for the normal observers had to be converted to $X_{\text {Judd }} Y_{\text {Judd }} Z_{\text {Judd }}$, which was done with the help of Vos's conversion formula and the necessary assumption that Judd's modified photopic luminous efficiency function $Y_{\text {Judd }}$ coincided with the CIE nonmodified function, that is, $Y=Y_{\text {Judd }}$. The new $L^{\prime} M^{\prime} S^{\prime}$ were converted back into $X^{\prime} Y^{\prime} Z^{\prime}$ using the inverse transformations, and the new representations in CIELAB and the volume calculation were computed.

For anomalous trichromats, the DeMarco et al. [33] data for the spectral sensitivities were used, and are representative of average anomalous trichromats. These sensitivities assume for the protanomalous a separation between the $M$ and $L^{\prime}$ cone pigments of $10 \mathrm{~nm}$ and for the deuteranomalous a separation between the $M^{\prime}$ and $L$ cone pigments of $6 \mathrm{~nm}$. Smith and Pokorny's fundamentals were used to convert anomalous cone excitations into tristimulus values of Judd. Vos's transformation was then applied to transform the data into CIE $1931 X Y Z$, and the representation in CIELAB color space and the volume calculation were computed.
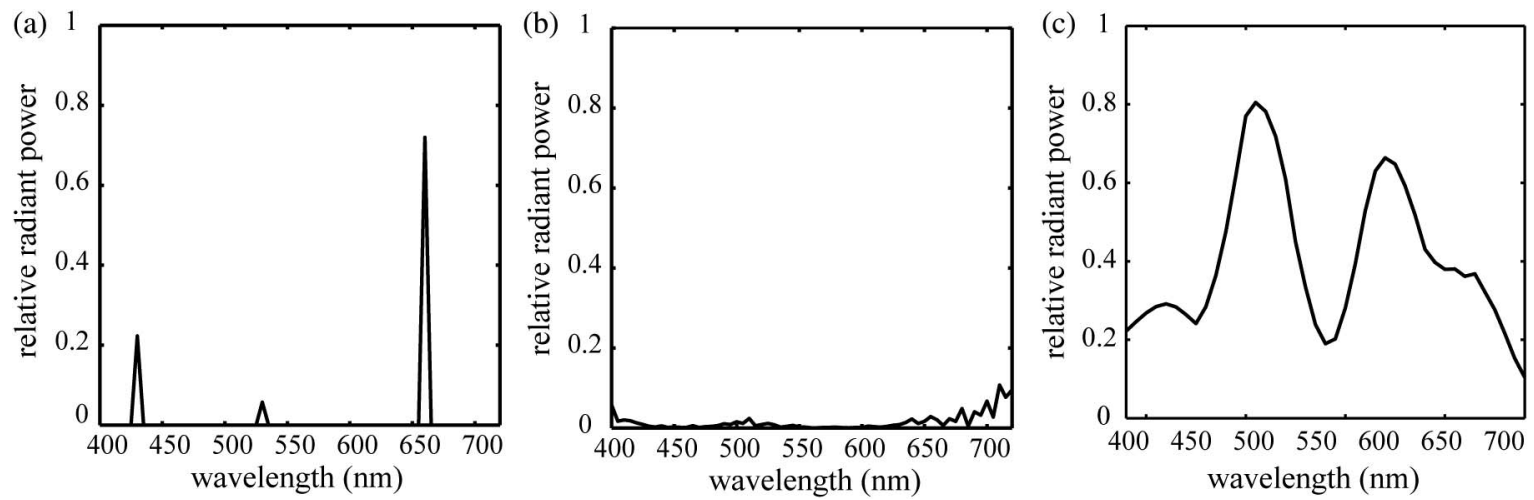

Fig. 1. (a) Relative radiant power distribution of the prime-color illuminant, (b) that derived by systematic probing with metamers at and around the Planckian locus, and (c) illuminant obtained here by spectral optimization. (All the spectra were normalized to the maximum value.) 


\section{Spectral Optimization}

The spectral optimization was implemented with an algorithm in Matlab based on the function fminsearch, which is based on the Nelder-Mead simplex method [34]. In the optimization, the dependent variable was the Rösch-MacAdam color volume, and the independent variable was the spectral radiance of the test illuminant from 400 to $720 \mathrm{~nm}$ in $10 \mathrm{~nm}$ steps. Several initial spectra were selected for the optimization procedure: the CIE illuminants [28] D65, E, FL3.7, and HP1. These spectra selected are representative of all the illuminants standardized by the CIE to describe the colorimetric characteristics of a reflecting or transmitting material.

\section{RESULTS}

A. Normal Observer

1. Rösch-MacAdam Volumes for Daylights and CIE Illuminants

To compare the discriminability obtained with typical illuminants with that obtained with illuminants with high-colordiscrimination capability, the Rösch-MacAdam volumes obtained with daylights with CCT in the range of 4000$25,000 \mathrm{~K}$ were computed. A similar exercise was carried out for the other CIE illuminants described in Section 2. The results for daylights are shown in Fig. 2. The volume increases monotonically with decreasing CCT up to a value of about $2.42 \times 10^{6}$. The variation of the volume across the range is about $10 \%$. The results for the other illuminants are shown in Table 1 . The volumes vary across illuminants but are all lower than the maximum obtained with daylight, at a CCT of $4000 \mathrm{~K}$.

\section{Rösch-MacAdam Volumes for Illuminants} with High-Color-Discrimination Capability

Figure 3 shows the results of the spectral optimization. In the upper row, Fig. 3(a) shows the spectrum of D65 for comparison, Fig. 3(b) shows the spectrally optimized illuminant obtained when the initial starting point of the optimization

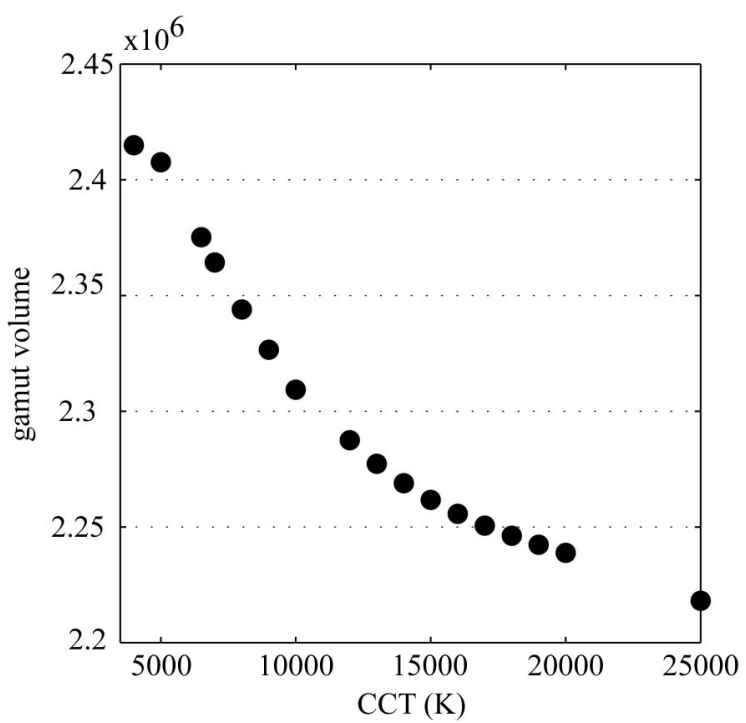

Fig. 2. Rösch-MacAdam volumes for daylight illuminants obtained for CCT in the range of 4000-25,000 K. Volumes are expressed in CIELAB space.
Table 1. Rösch-MacAdam Volumes Obtained with Several Standard Illuminants and Three-SpectralBands Illuminants for the Normal Observer ${ }^{a}$

\begin{tabular}{lc}
\hline Illuminant & Rösch-MacAdam Volumes $\left(\times 10^{6}\right)$ \\
\hline A & 2.33 \\
C & 2.34 \\
D65 & 2.37 \\
E & 2.38 \\
F11 & 2.19 \\
FL3.7 & 2.02 \\
HP1 & 1.49 \\
Prime color 1973 & 1.99 \\
Masuda and Nascimento's & 3.02 \\
Spectrally optimized & 2.79 \\
\hline
\end{tabular}

${ }^{a}$ Volumes are expressed in CIELAB space.

was D65, and Fig. 3(c) shows the average spectrally optimized illuminant obtained from a set of different starting illuminants. The corresponding color volumes are also shown in the bottom row. The chromaticity of the spectrum in Fig. 3(c) expressed in the CIE 1931 space was $(0.316,0.406)$. The optimized spectra obtained had three peaks, around 420, 520 , and $640 \mathrm{~nm}$, and did not vary much with the departure point of the optimization procedure. Therefore, the spectrally optimized illuminant was assumed to be the average of the spectrally optimized illuminant obtained from a set of different starting illuminants [Fig. $\underline{3(\mathrm{c})}$ ]. Table $\underline{1}$ shows the RöschMacAdam volumes of the spectrally optimized illuminant obtained here [spectrum represented on Fig. 1(c)], of the illuminant derived by Masuda and Nascimento [27], and of the prime-color illuminant. Unlike the prime-color illuminant, the other two illuminants show a volume that is considerably larger than the maximum produced by daylight. Figure $\underline{4}$ shows the spectral profile of the three-band illuminants: (a) prime color, (b) Masuda and Nascimento's, and (c) spectrally optimized, and the corresponding Rösch-MacAdam color solid. The comparison between daylight and the prime-color illuminant was carried out in the analysis reported in [27].

\section{Visual Effects of Illuminants with High-Color-} Discrimination Capability

To illustrate the visual effects of these high-colordiscrimination illuminants, Fig. $\underline{5}$ represents four complex scenes illuminated by the equi-energy illuminant $\mathrm{E}$ and by each of the high-color-discrimination illuminants with corresponding color solid volume. This volume was estimated for each scene by segmenting the corresponding CIELAB color volume into cubes of unitary side and by counting the number of nonempty cubes. The images were generated from hyperspectral data acquired in the range of $400-720 \mathrm{~nm}$ at $10 \mathrm{~nm}$ intervals and later interpolated to $5 \mathrm{~nm}$ steps to better adjust the hyperspectral data to the spectral profile of the illuminants (for details on the spectral imaging technique, see [25]).

It can be seen from Fig. $\underline{5}$ that the chromatic diversity associated with illuminants with high-color-discrimination capability is larger than that observed with the illuminant E.

\section{B. Color-Deficient Observer}

Table 2 shows the Rösch-MacAdam volumes for the color-deficient observers estimated for each of the three 


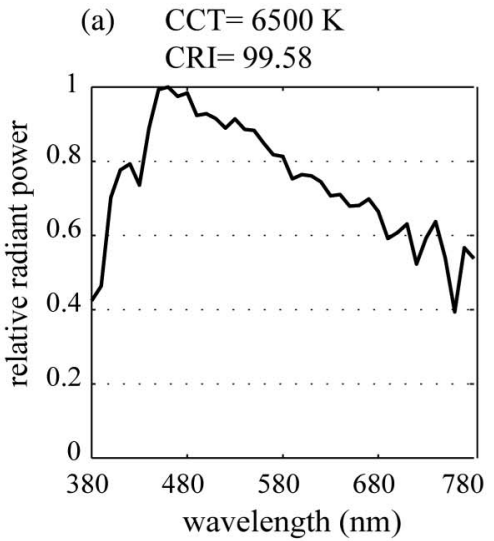

(b) $\mathrm{CCT}=6920 \mathrm{~K}$

$\mathrm{CRI}=59.23$

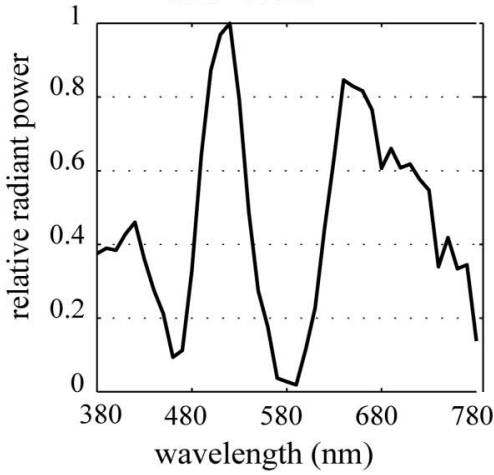

(e)
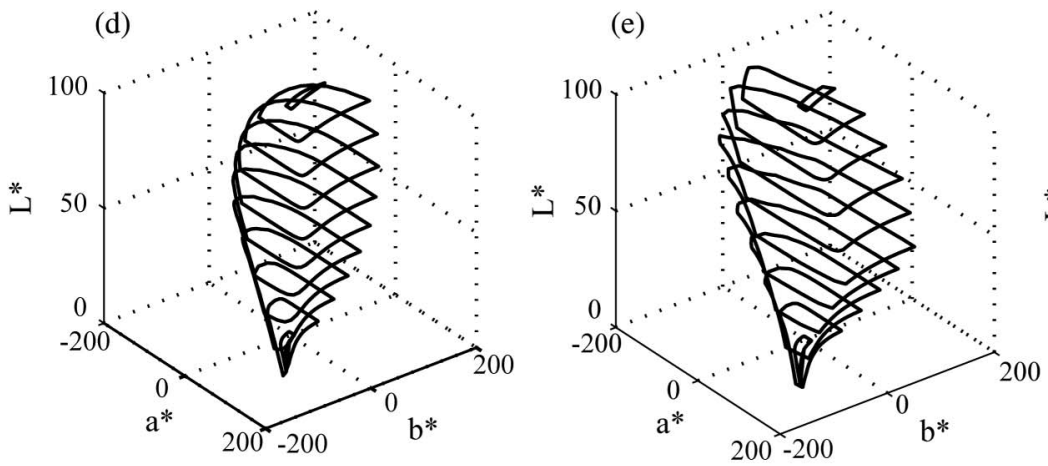

(c) $\mathrm{CCT}=6092 \mathrm{~K}$

$\mathrm{CRI}=73.50$
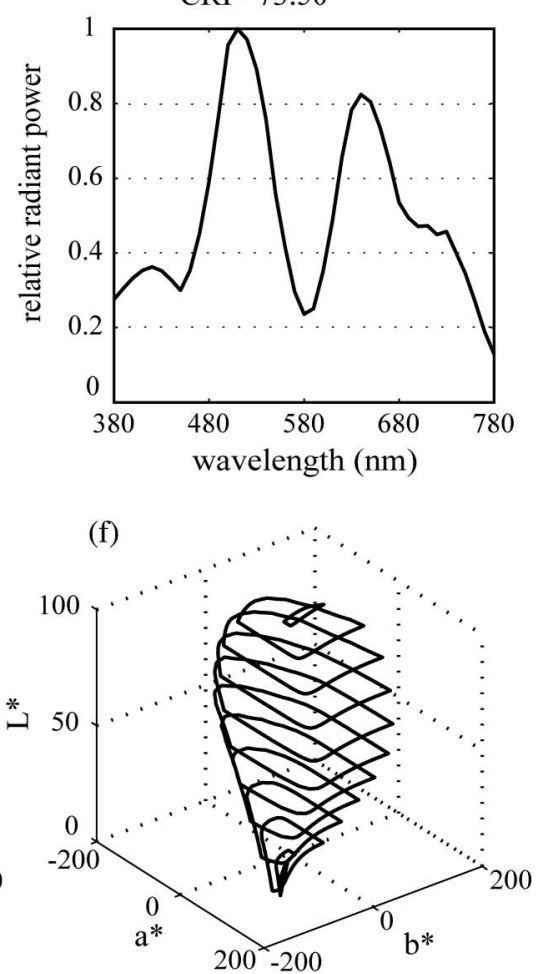

Fig. 3. Upper row shows (a) relative radiant power distribution of the illuminant D65, (b) that of the optimized illuminant obtained with D65 as the initial illuminant in the computational procedure, and (c) illuminant obtained by averaging from different initial illuminants in the computational procedure. The bottom row shows (d) Rösch-MacAdam color solids represented in the CIELAB color space under illuminant D65, (e) optimized illuminant, and (f) average optimized illuminant. These volumes are $2.375 \times 10^{6}, 2.992 \times 10^{6}$, and $2.791 \times 10^{6}$, respectively.
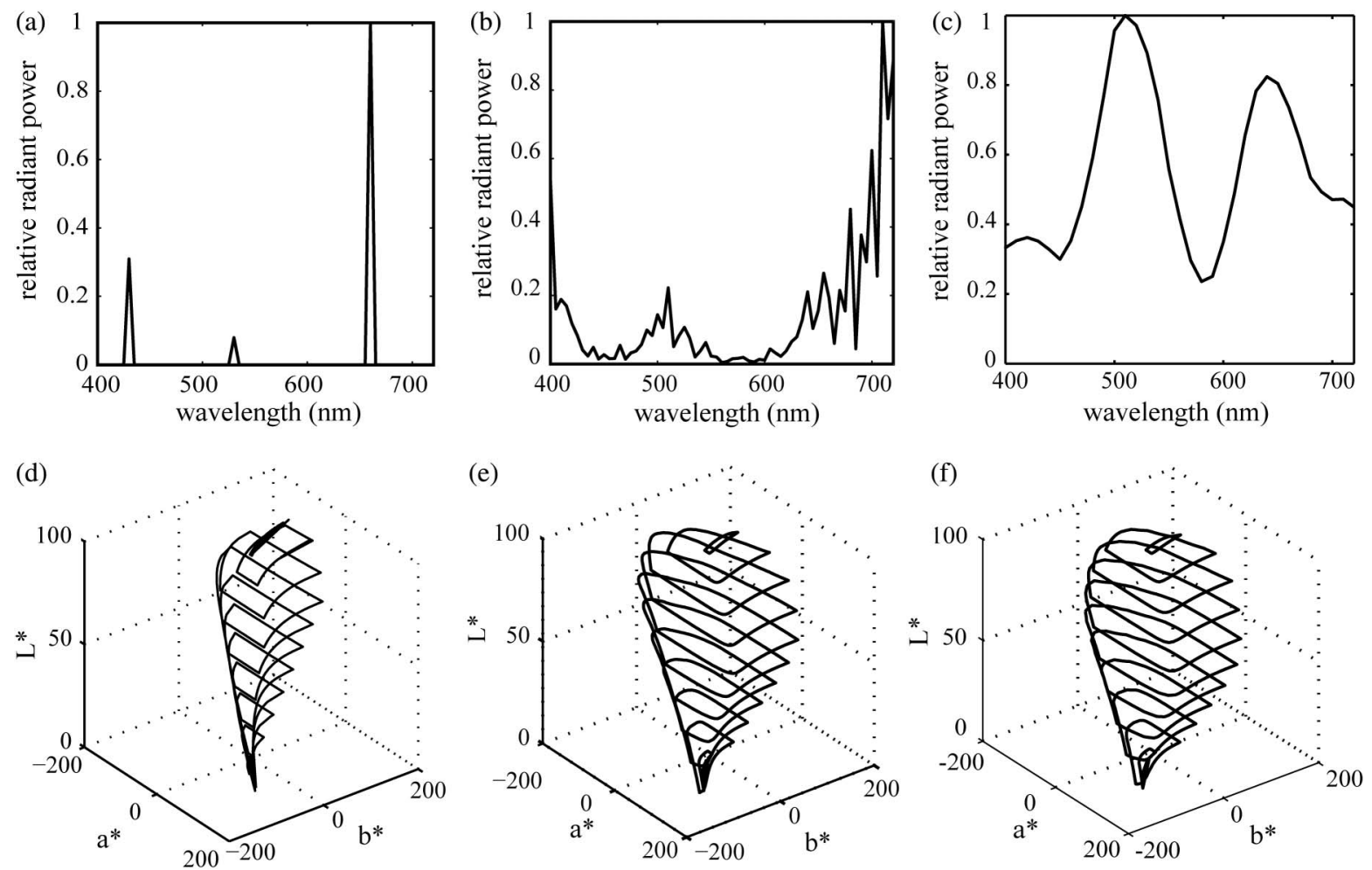

Fig. 4. Upper row shows (a) relative radiant power distribution of the prime-color illuminant, (b) Masuda and Nascimento's illuminant, and (c) spectrally optimized illuminant. The bottom row shows the Rösch-MacAdam color solids represented in the CIELAB color space under (d) prime-color illuminant, (e) Masuda and Nascimento's illuminant, and (f) spectrally optimized illuminant. These volumes are $1.99 \times 10^{6}$, $3.02 \times 10^{6}$, and $2.79 \times 10^{6}$, respectively. 


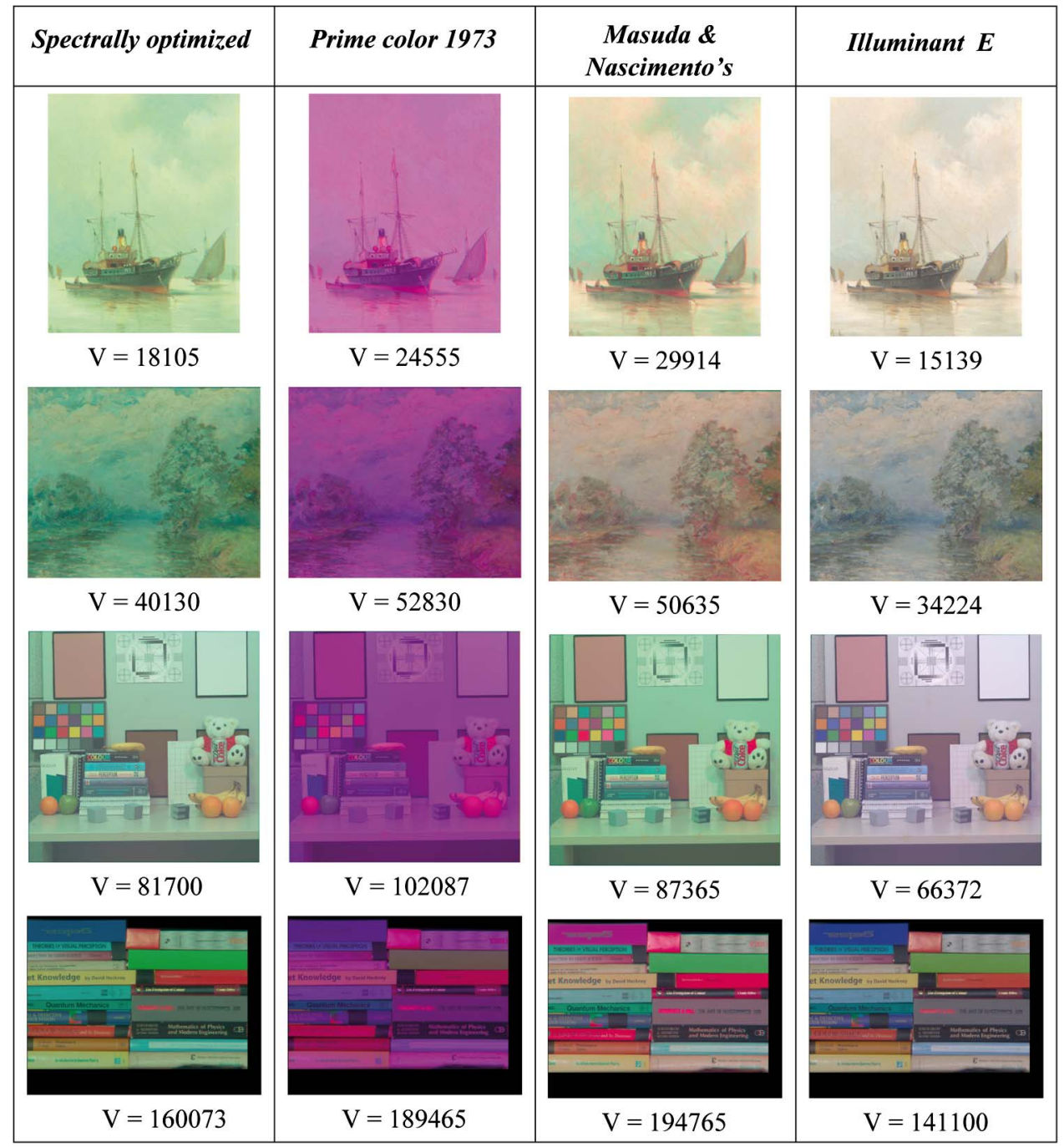

Fig. 5. Visual effects of the high-color-discrimination illuminants on artistic paintings and indoor scenes. Also represented is the corresponding color solid volume (V) for each tested illuminant.

high-color-discrimination illuminants and for the illuminant $\mathrm{E}$, the one producing the largest volume of Table 1 . For dichromats, only one of the high-color-discrimination illuminants, the spectrally optimized, outperforms the illuminant $\mathrm{E}$ : in tritanopia its volume is larger than that produced by the illuminant $\mathrm{E}$ by about $2 \%$. For anomalous trichromacy, all three high-color-discrimination illuminants performed better than illuminants $\mathrm{E}$ for the deuteranomalous case, but for the protanomalous case this happens only with the illuminant optimized here. The largest enhancement is obtained by
Masuda and Nascimento's illuminant for the deuteranomalous case, about $30 \%$. This illuminant is, however, not so good for the protanomalous case, and is particularly bad for the dichromats. The worst illuminant is the prime color, as it represents impairments larger than $45 \%$ for the dichromats.

Figure 6 shows the Rösch-MacAdam volumes for the classes of color deficiencies and for the best high-colordiscrimination illuminant, the spectrally optimized one derived here, and those for illuminant $\mathrm{E}$ for comparison.

Table 2. Rösch-MacAdam Volumes for Color-Deficient Observers for Each of the High-Color-Discrimination Illuminants and for Illuminant $\mathbf{E}$ for Comparison ${ }^{a}$

\begin{tabular}{|c|c|c|c|c|}
\hline & Spectrally Optimized & Prime Color & Masuda and Nascimento's & Illuminant $\mathrm{E}$ \\
\hline Tritanope & 18,020 & 9235 & 10,879 & 17,626 \\
\hline Deuteranope & 15,205 & 7043 & 12,142 & 16,036 \\
\hline Protanope & 15,514 & 3568 & 9211 & 16,093 \\
\hline Deuteranomalous & $1.82 \times 10^{6}$ & $1.71 \times 10^{6}$ & $2.06 \times 10^{6}$ & $1.69 \times 10^{6}$ \\
\hline Protanomalous & $1.88 \times 10^{6}$ & $1.33 \times 10^{6}$ & $1.48 \times 10^{6}$ & $1.82 \times 10^{6}$ \\
\hline
\end{tabular}

${ }^{a}$ Volumes are expressed in CIELAB color space. 
(a) deuteranomalous

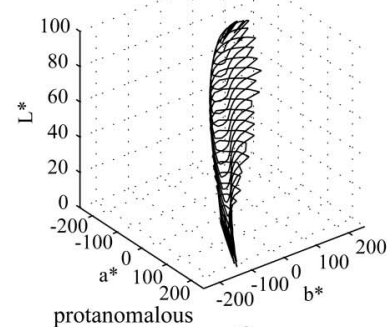

protanomalous

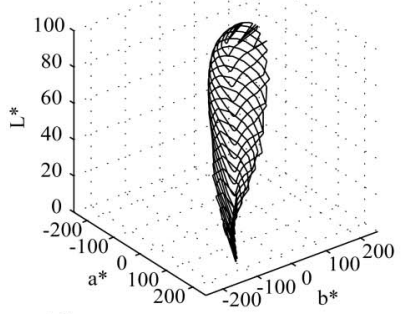

$$
\text { tritanope }
$$

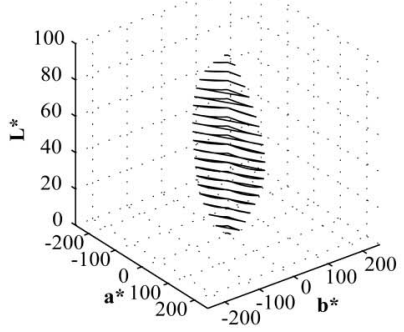

deuteranope
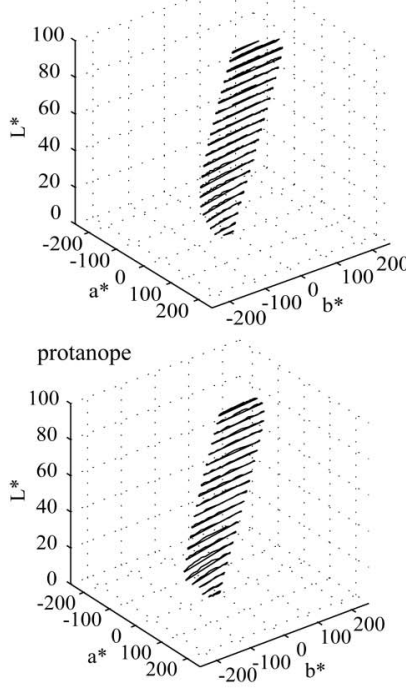

(b) deuteranomalous
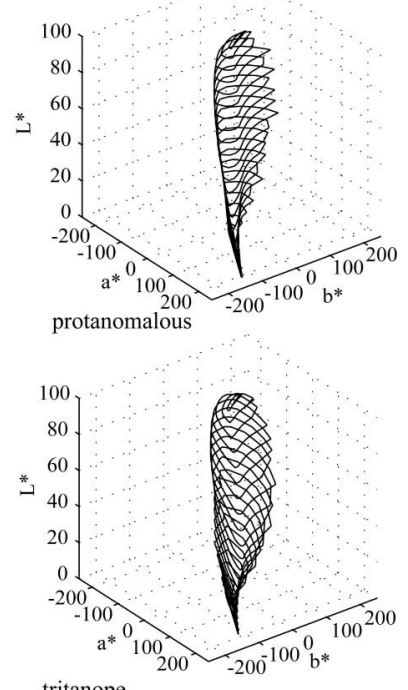

tritanope
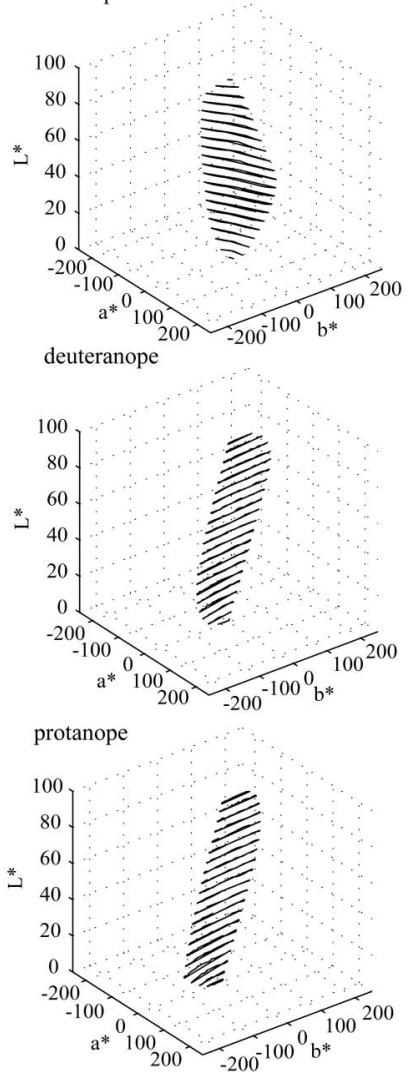

Fig. 6. Rösch-MacAdam color solids represented in the CIELAB color space under (a) illuminant $\mathrm{E}$ and (b) optimized illuminant.

\section{DISCUSSION AND CONCLUSION}

The illuminants with three spectral bands with high-colordiscrimination capability studied here have variable effects on chromatic diversity perceived by color-deficient observers. The prime-color illuminant and the illuminant derived by Masuda and Nascimento (2012) only improve the discrimination for deuteranomalous but not for protanomalous or dichromats. The prime color is particularly bad with dichromats. The illuminant derived here by spectral optimization was revealed to be better: it clearly improves discrimination for red-green anomalies, and it is almost neutral for dichromats. We conclude that the hypothesis put forward by Thornton that high-color-discrimination illuminants also enhance discrimination for abnormal observers [7] is only true in some cases-namely, for the spectrally optimized illuminant derived here, but clearly not for the prime-color illuminant.

The results presented here were obtained by representing the chromatic data in the CIELAB color space, a color space with known nonuniformities. But as the relevant estimates here are relationships rather than absolute values, these nonuniformities may have a small impact on the conclusions.

The estimations here were based on models of the perception of color-deficient observers. For dichromats, the model proposed is likely to be an oversimplification as there is evidence that dichromats may experience larger chromatic diversity than assumed here, e.g., large-field trichromacy [35,36]. For anomalous observers, the assumption is that the color vision system of these observers is the same as the normal observer except for the spectral sensitivities of the cone photoreceptors. Although this seems reasonable, it is not yet demonstrated experimentally. Also, the anomalies occur within an extended range, and what is considered here is just the average anomalous observer as reported by DeMarco et al. [33]. In spite of these approximations and incertitude, the exercise carried out here is probably the best possible with the available tools, and it is likely to serve as a guide for efficient illumination for normal and color-deficient observers.

\section{ACKNOWLEDGMENTS}

This work was supported by the European Regional Development Fund through Program COMPETE (FCOMP-01-0124FEDER-009858), by National Portuguese funds through Fundação para a Ciência e a Tecnologia (grant PTDC/EEAEEL/098572/2008), and by the European Union and Spanish Ministry of Economy and Competitiveness under grant DPI2011-30090-C02-02.

\section{REFERENCES}

1. D. H. Foster, "Color constancy," Vis. Res. 51, 674-700 (2011).

2. E. Mahler, J. J. Ezrati, and F. Vienot, "Testing LED lighting for colour discrimination and colour rendering," Color Res. Appl. 34, 8-17 (2009).

3. M. P. Royer, K. W. Houser, and A. M. Wilkerson, "Color discrimination capability under highly structured spectra," Color Res. Appl. 37, 441-449 (2012).

4. X. Guo and K. W. Houser, "A review of colour rendering indices and their application to commercial light sources," Lighting Res. Technol. 36, 183-199 (2004).

5. W. A. Thornton, "Color-rendering capability of commercial lamps,” Appl. Opt. 11, 1078-1086 (1972).

6. W. A. Thornton, "Fluorescent lamps with high colordiscrimination capability,” J. Illum. Eng. Soc. 3, 61-64 (1973).

7. W. A. Thornton, "Colour discrimination enhancement by the illuminant," in Second Symposium of the International Research Group on Colour Vision Deficiencies (Karger, 1974), pp. 312-313.

8. W. A. Thornton, "Color-discrimination index," J. Opt. Soc. Am. 62, 191-194 (1972).

9. J. M. M. Linhares and S. M. C. Nascimento, "A chromatic diversity index based on complex scenes," J. Opt. Soc. Am. A 29, A174-A181 (2012).

10. M. S. Rea and J. P. Freyssinier-Nova, "Color rendering: a tale of two metrics," Color Res. Appl. 33, 192-202 (2008).

11. H. Xu, "Color-rendering capacity of light," Color Res. Appl. 18, 267-269 (1993). 
12. G. Wyszecki and W. S. Stiles, Color Science: Concepts and Methods, Quantitative Data and Formulae, 2nd ed. (Wiley, 1982).

13. R. Luther, "Aus dem Gebiet der Farbreizmetrik," Z. Tech. Phys. 8, 540-558 (1927).

14. N. D. Nyberg, "Zum Aufbau des Farbenkörpers im Raume aller Lichtempfindungen," Z. Phys. A 52, 406-419 (1929).

15. D. L. Macadam, "Theory of the maximum visual efficiency of colored materials," J. Opt. Soc. Am. 25, 249-252 (1935).

16. D. L. Macadam, "Maximum visual efficiency of colored materials," J. Opt. Soc. Am. 25, 316-367 (1935).

17. F. Martínez-Verdú, E. Perales, E. Chorro, D. D. de Fez, V. Viqueir, and E. Gilabert, "Computation and visualization of the MacAdam limits for any lightness, hue angle, and light source," J. Opt. Soc. Am. A 24, 1501-1515 (2007).

18. K. Masaoka, "Fast and accurate model for optimal color computation," Opt. Lett. 35, 2031-2033 (2010).

19. C. Li, M. R. Luo, M.-S. Cho, and J.-S. Kim, "Linear programming method for computing the gamut of object color solid," J. Opt. Soc. Am. A 27, 985-991 (2010).

20. E. Perales, F. Martínez-Verdú, J. M. Linhares, and S. M. C. Nascimento, "Number of discernible colors for color-deficient observers estimated from the MacAdam limits," J. Opt. Soc. Am. A 27, 2106-2114 (2010).

21. J. M. Linhares, P. D. Pinto, and S. M. Nascimento, "The number of discernible colors in natural scenes," J. Opt. Soc. Am. A 25, 2918-2924 (2008)

22. M. R. Pointer and G. G. Attridge, "The number of discernible colours," Color Res. Appl. 23, 52-54 (1998).

23. W. A. Thornton, "The prime colors of human vision: their prescription for illumination, color printing, color photography, color television, and visual clarity," in The First European Conference on Color in Graphics, Imaging and Vision (CGIV), Poitiers, France, 2002, pp. 56-60.

24. J. M. Linhares, P. D. Pinto, and S. M. Nascimento, "Color rendering of art paintings under CIE illuminants for normal and color deficient observers," J. Opt. Soc. Am. A 26, 1668-1677 (2009).
25. J. M. M. Linhares, P. E. R. Felgueiras, P. D. Pinto, and S. M. C. Nascimento, "Colour rendering of indoor lighting with CIE illuminants and white LEDs for normal and colour deficient observers," Ophthalmic Physiolog. Opt. 30, 618-625 (2010).

26. J. M. M. Linhares, P. D. Pinto, and S. M. C. Nascimento, "The number of discernible colors perceived by dichromats in natural scenes and the effects of colored lenses," Vis. Neurosci. 25, 493-499 (2008).

27. O. Masuda and S. M. C. Nascimento, "Lighting spectrum to maximize colorfulness," Opt. Lett. 37, 407-409 (2012).

28. CIE, Colorimetry, CIE Publ 15:2004 (CIE, 2004).

29. D. B. Judd, D. L. MacAdam, G. Wyszecki, H. W. Budde, H. R. Condit, S. T. Henderson, and J. L. Simonds, "Spectral distribution of typical daylight as a function of correlated color temperature," J. Opt. Soc. Am. 54, 1031-1040 (1964).

30. C. B. Barber, D. P. Dobkin, and H. Huhdanpaa, "The Quickhull algorithm for convex hulls," ACM Trans. Math. Softw. 22, 469-483 (1996).

31. H. Brettel, F. Vienot, and J. D. Mollon, "Computerized simulation of color appearance for dichromats," J. Opt. Soc. Am. A 14, 2647-2655 (1997).

32. V. C. Smith and J. Pokorny, "Spectral sensitivity of color-blind observers and the cone photopigments," Vis. Res. 12, 2059-2071 (1972).

33. P. DeMarco, J. Pokorny, and V. C. Smith, "Full-spectrum cone sensitivity functions for X-chromosome-linked anomalous trichromates," J. Opt. Soc. Am. A 9, 1465-1476 (1992).

34. J. C. Lagarias, J. A. Reeds, M. H. Wright, and P. E. Wright, "Convergence properties of the Nelder-Mead simplex method in low dimensions," SIAM J. Optim. 9, 112-147 (1998).

35. A. L. Nagy and R. M. Boynton, "Large-field color naming of dichromates with rods bleached," J. Opt. Soc. Am. 69, 1259-1265 (1979).

36. V. C. Smith and J. Pokorny, "Large-field trichromacy in protanopes and deuteranopes.," J. Opt. Soc. Am. 67, 213-220 (1977) 